\title{
ULTRA-SHORT PERIOD BINARIES FROM THE CATALINA SURVEYS
}

\author{
A. J. Drake ${ }^{1}$, S. G. Djorgovski ${ }^{1}$, D. García-Áldvarez ${ }^{2,3,4}$, M. J. Graham $^{1}$, M. Catelan ${ }^{5}$, A. A. Mahabal ${ }^{1}$, C. Donalek ${ }^{1}$, \\ J. L. Prieto ${ }^{6}$, G. Torrealba ${ }^{5}$, S. Abraham ${ }^{7}$, R. Williams $^{1}$, S. Larson $^{8}$, and E. Christensen ${ }^{8}$ \\ ${ }^{1}$ California Institute of Technology, 1200 East California Boulevard, CA 91225, USA \\ 2 Instituto de Astrofísica de Canarias, Avenida Vía Láctea, 38205 La Laguna, Tenerife, Spain \\ ${ }^{3}$ Departamento de Astrofísica, Universidad de La Laguna, 38205 La Laguna, Tenerife, Spain \\ ${ }^{4}$ Grantecan S. A., Centro de Astrofísica de La Palma, Cuesta de San José, 38712 Breña Baja, La Palma, Spain \\ ${ }_{5}^{5}$ Pontificia Universidad Católica de Chile, Departamento de Astronomía y Astrofísica, Facultad de Física, \\ Av. Vicuña Mackena 4860, 782-0436 Macul, Santiago, Chile \\ ${ }^{6}$ Department of Astronomy, Princeton University, 4 Ivy Lane, Princeton, NJ 08544, USA \\ ${ }^{7}$ St. Thomas College, Kozhencheri 689641, India \\ ${ }^{8}$ Department of Planetary Sciences, The University of Arizona, Lunar and Planetary Laboratory, 1629 East University Boulevard, Tucson, AZ 85721, USA \\ Received 2014 April 4; accepted 2014 June 12; published 2014 July 17
}

\begin{abstract}
We investigate the properties of 367 ultra-short period binary candidates selected from 31,000 sources recently identified from Catalina Surveys data. Based on light curve morphology, along with WISE, Sloan Digital Sky Survey, and GALEX multi-color photometry, we identify two distinct groups of binaries with periods below the 0.22 day contact binary minimum. In contrast to most recent work, we spectroscopically confirm the existence of $\mathrm{M}$ dwarf $+\mathrm{M}$ dwarf contact binary systems. By measuring the radial velocity variations for five of the shortestperiod systems, we find examples of rare cool white dwarf (WD)+M dwarf binaries. Only a few such systems are currently known. Unlike warmer WD systems, their UV flux and optical colors and spectra are dominated by the M-dwarf companion. We contrast our discoveries with previous photometrically selected ultra-short period contact binary candidates and highlight the ongoing need for confirmation using spectra and associated radial velocity measurements. Overall, our analysis increases the number of ultra-short period contact binary candidates by more than an order of magnitude.
\end{abstract}

Key words: galaxies: stellar content - Galaxy: stellar content - stars: variables: general

Online-only material: color figures

\section{INTRODUCTION}

The study of eclipsing binaries provides the opportunity of determining stellar parameters with a high degree of accuracy using constraints on the geometry and motions of the system (Southworth 2012, and references therein). Amongst other things, eclipsing binaries can be used to probe various stages of stellar evolution via the determination of system parameters such as the orbital period, inclination, radii, and masses.

The types of eclipsing binaries are generally defined by the degree of separation of the components. These include overcontact, contact, semi-detached, and detached systems that can often be discerned from light curve shapes. Eclipsing contact binaries are referred to as W Ursae Majoris (W UMa's) stars (or EW variable types). Such systems allow mass to flow from one star to the other and both stars usually have similar temperatures and types. Here, the depth of the eclipse is most dependent on the system geometry; in contrast, the depth of the eclipse reflects the relative effect temperatures in detached binaries (Rucinski 2001). Nevertheless, slight differences in the eclipse depth still occur in contact systems due to differences in the temperature of the component stars.

For many years, it has been suspected that there is a minimum period for contact binaries at $P \sim 0.22$ day (Rucinski 1992, 1997). Numerous explanations have been put forth to explain the observed abrupt cut off. For example, Stepien (2006) proposed that the limit was due to the decrease in the efficiency of angular momentum loss with decreasing mass on the main sequence. In such cases, the current age of the systems comprising the binary population produces the limit. More recently, Jiang et al. (2012) investigated mass transfer in low-mass binaries and found this to be unstable. They suggest that systems with the lowest mass might quickly coalesce, leading to their observational absence.

In the past few years, increasing numbers of eclipsing binary candidates have been discovered with periods of less than 0.22 day. For example, the detached binary OGLE-BW03V038 was discovered with a period of 0.1984 day by Maceroni \& Rucinski (1997), and a similar detached eclipsing binary system with main-sequence stars was found with a period of 0.1926 day by Norton et al. (2007) and confirmed by Dimitrov \& Kjurkchieva (2010). However, as detached systems are not in the process of mass transfer, they exist in different evolutionary state than contact systems.

Recently, Norton et al. (2011) identified 14 new eclipsing systems with periods $P<0.22$ day among 30 million SuperWASP sources. Although the periods of two of these systems were later found to be longer (Lohr et al. 2012). The number of sub-0.2 days Super WASP systems was increased to 49 by Lohr et al. (2013). Their results include a number of possible contact systems with periods in the 0.2-0.22 day range. Similarly, Nefs et al. (2012) discovered 14 eclipsing binary candidates with periods of less than 0.22 day. Among these systems, Nefs et al. (2012) spectroscopically confirm a detached system with a 0.18 day period containing an $\mathrm{M}$ dwarf. However, they did not attempt to measure radial velocities. In contrast, Davenport et al. (2013) discovered a likely contact binary system with a period of 0.19856 day. They confirm the presence of a M dwarf and find radial velocity variations that are consistent binary $\mathrm{M}$ dwarf system. Nevertheless, despite the increasing evidence for short period contact binary systems below the 0.22 day period 


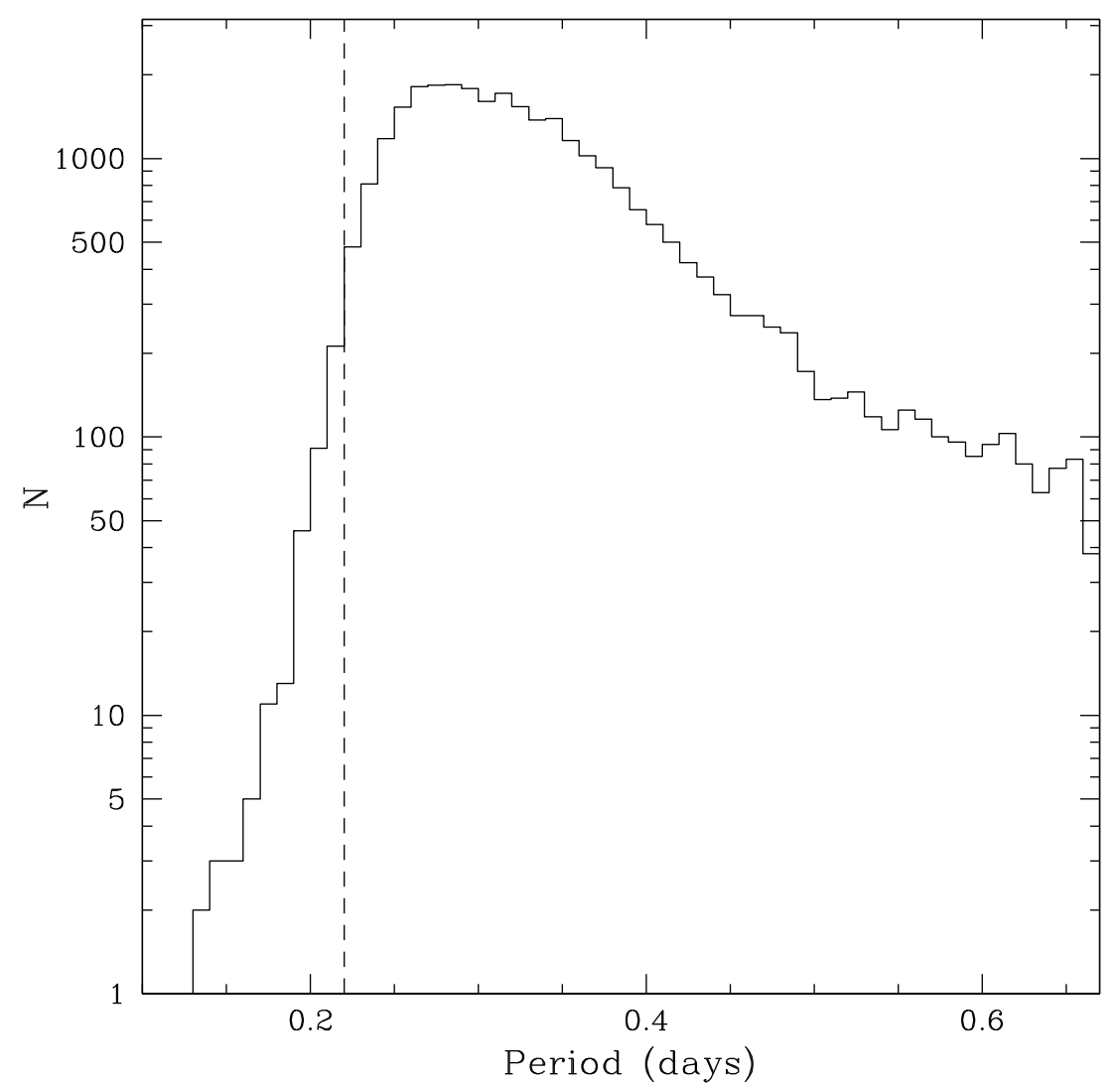

Figure 1. Period distribution of contact binaries. The solid line give the distribution of contact binary candidates from CSDR1. The dashed line denotes the location of the purported 0.22 day contact binary period minimum.

cutoff, only a handful of candidates have so far been spectroscopically confirmed, and fewer still have had their component masses determined via radial velocities measurements.

In this analysis, we will investigate both the spectral types and radial velocities for ultra-short period binary systems discovered during the compilation of the Catalina Surveys periodic variable stars catalog (Drake et al. 2014).

\section{OBSERVATIONAL DATA}

The Catalina Sky Survey (CSS $)^{9}$ uses three telescopes to cover the sky between declination $\delta=-75^{\circ}$ and $+65^{\circ}$ in order to discover near Earth objects and potential hazardous asteroids. Catalina observations do not cover regions within $15^{\circ}$ of the Galactic plane because of blending in crowded stellar regions. All the images are taken unfiltered, and photometry is carried out using the aperture photometry program SExtractor (Bertin \& Arnouts 1996). These measurements are transformed to an approximate $V$ magnitude ( $V_{\mathrm{CSS}}$ ) using fiducial Two Micron All Sky Survey color-selected G dwarf stars as outlined in Drake et al. (2013).

In this paper, we select and analyze the ultra-short period systems $(P<0.22$ day) among the 31,000 contact binary candidates discovered by Drake et al. (2014) from data in the Catalina Data Release 1 (CSDR1; Drake et al. 2012). The CSDR1 data set itself consists only of data taken with the Catalina Schmidt Survey telescope. This includes an average of 250 observations per position spanning six years for 198 million discrete sources. The CSDR1 sources have $12<V<20$, and lie in the region $0^{\circ}<\alpha<360^{\circ}$ and $-30^{\circ}<\delta<65^{\circ}$. The light

\footnotetext{
9 http://www.lpl.arizona.edu/css/
}

curve data for all CSDR1 sources (including those presented here) are publicly available online. ${ }^{10}$

\section{SHORT PERIOD BINARIES}

The Drake et al. (2014) variable catalog contains 367 systems with periods less than 0.22 day; of these, 73 have periods less than 0.2 day. The eclipsing systems were selected based on the morphological classification from among 61,000 periodic variables in the catalog. Additional confirmation of the variable classifications in the catalog was performed by Drake et al. (2014) using color information derived by combining CSS, WISE, and Sloan Digital Sky Survey (SDSS) photometry. Spectroscopic parameters were also derived from SDSS data and confirm the separation between the eclipsing binaries and pulsating stars such as RR Lyrae stars. As the photometry spans many years, we do not expect contamination by spotted variable stars (such as RS Canum Venaticorum variables), since such objects exhibit clear changes in the average brightness, amplitude, and phase of flux variations (e.g., see Drake 2006, Figure 2; Drake et al. 2014, Figure 18).

In Figure 1, we present the period distribution of the 31,000 (Drake et al. 2014) contact binary candidates. Here we see there is indeed a very sharp decline in the number of short period systems below $\sim 0.27$ day. Given the large number of contact systems in CSDR1, the fraction of ultra-short period objects amounts to only $\sim 0.26 \%$ of the total number. This result in itself very strongly supports the scarcity of short period systems. However, we note that past surveys for such systems have had a clear observational bias against finding such systems. That is to

\footnotetext{
$10 \mathrm{http}: / /$ catalinadata.org
} 

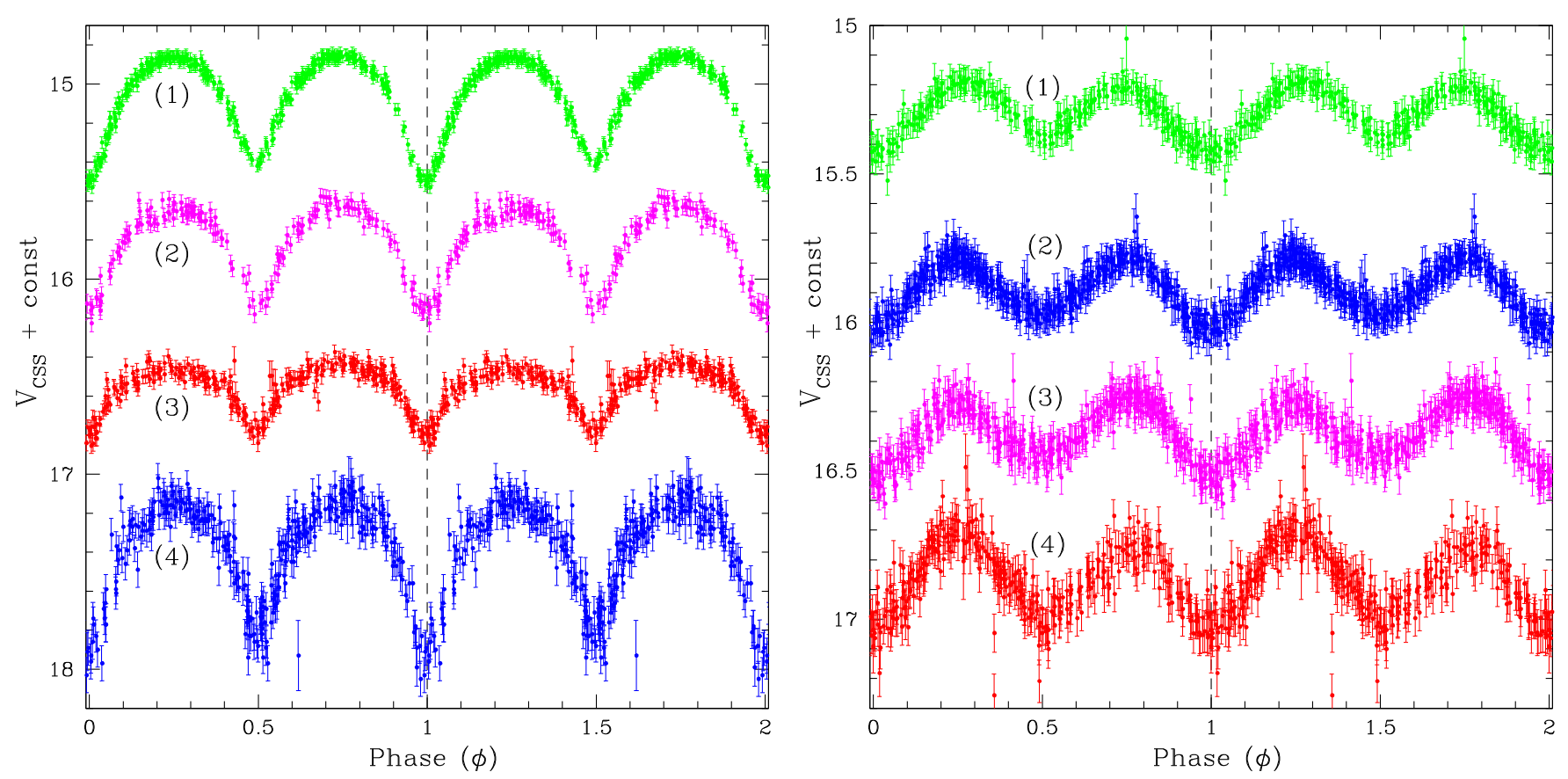

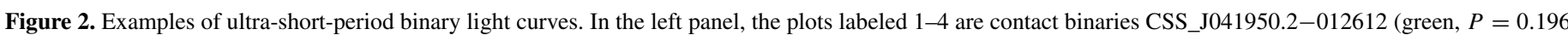

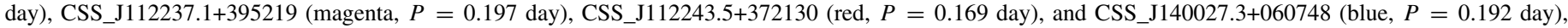

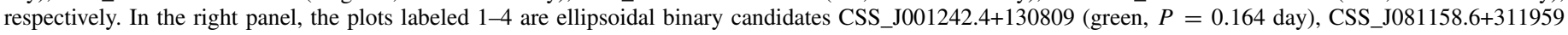
(blue, $P=0.156$ day), CSS_J090119.2+114254 (magenta, $P=0.187$ day), and CSS_J111647.8 +294603 (red, $P=0.146$ day), respectively.

(A color version of this figure is available in the online journal.)

say, the stars that comprise short-period systems are much fainter than the $\mathrm{dG}$ and $\mathrm{dK}$ stars that make up longer-period systems. For example, in the $V$ band, an MOV star is 4.4 mag fainter than a G0V star. Thus, shallow wide-field synoptic surveys such as ASAS (Pojmanski 1997) that only reached $V=13$ have probed a very small volume of potential M-dwarf binaries compared to CSDR1 (e.g., see Rucinski 2006, Figure 4).

In order to investigate the nature of the ultra-short period sources we re-examined the light curves of all the objects Drake et al. (2014) identified as eclipsing contact and ellipsoidal binary candidates below the nominal ultra-short period limit ( 0.22 day). We observed a clear division in the morphology of the source light curves. Approximately half exhibited the W-shaped light curves expected for W UMa contact binaries, while the other half exhibited much more sinusoidal light curves, as seen in over-contact systems where the stars share an outer envelope and both exceed their Roche limit.

Although over-contact, contact, semi-detached, and detached binaries can exhibit quite distinct light curve shapes, they appear similar at the limit between these types of binary systems or, alternatively, when they are observed with low signal to noise. However, in this analysis, we found that the light curves for short-period binaries were generally quite well separated into just two groups. In Figure 2, we contrast the two main types of short-period light curves.

This same shape division can also be seen in the light curves of the short period binary candidates presented by Nefs et al. (2012). However, the light curves of ellipsoidal variables closely resemble those of over-contact binaries. For ellipsoidal variables, the variation is due to the distorted shapes of a star or stars. This can occur even when the objects are far from contact. Thus the sinusoidal light curves of some of the (Nefs et al. 2012) short-period contact binary candidates are consistent with both over-contact binaries and the ellipsoidal variations due to compact companions such white dwarfs (WDs) or subdwarfs. Such short-period sinusoidal light curves have been found in many WD main-sequence (WDMS) systems (e.g., RodriguezGil et al. 2009; Pyrzas et al. 2012; Parsons et al. 2013). In fact, systems with such light curves were specifically rejected from the short-period main-sequence contact binary sample of Norton et al. (2011) because of the possible contamination of WDMS systems.

For ellipsoidal variables, the amplitude is generally limited to $\sim 0.3 \mathrm{mag}$, since the variations are due to the distorted star filling its Roche lobe (Parsons et al. 2013). At very short periods, a distorted star will overflow its Roche lobe becoming a cataclysmic variable. The accretion induced variability in $\mathrm{CV}$ systems generally distinguishes them from stable non-accreting WDMS systems.

To investigate the nature of the objects with short-period objects, we looked at the colors of sources in the two groups using WISE, SDSS, and GALEX data. In Figure 3, we show the WISE data for the eclipsing binaries as well as $\delta$ Scuti stars (that have similar periods). The separation between the pulsating $\delta$ Scuti stars and the eclipsing and ellipsoidal-like ones is quite clear. However, some of the ellipsoidal candidates do have colors similar to $\delta$ Scutis. These objects may either be systems where the companion is a hot WD or an incorrectly classified $\delta$ Scutis. There is clearly significant overlap between ellipsoidal candidates and eclipsing systems. There are also ellipsoidal candidates that have $V-w 1>3.5$. These objects are redder than most of the eclipsing objects and thus suggest late stellar types.

In Figure 4, we show the $u-g$ and $i-z$ versus $g-r$ plots for the contact eclipsing binaries from Drake et al. (2014) with SDSS DR9 photometry along with $\delta$ Scuti stars and spectroscopically identified WD+dM binaries from the RebassaMansergas et al. (2012) catalog. We also plot the WD+dM model 


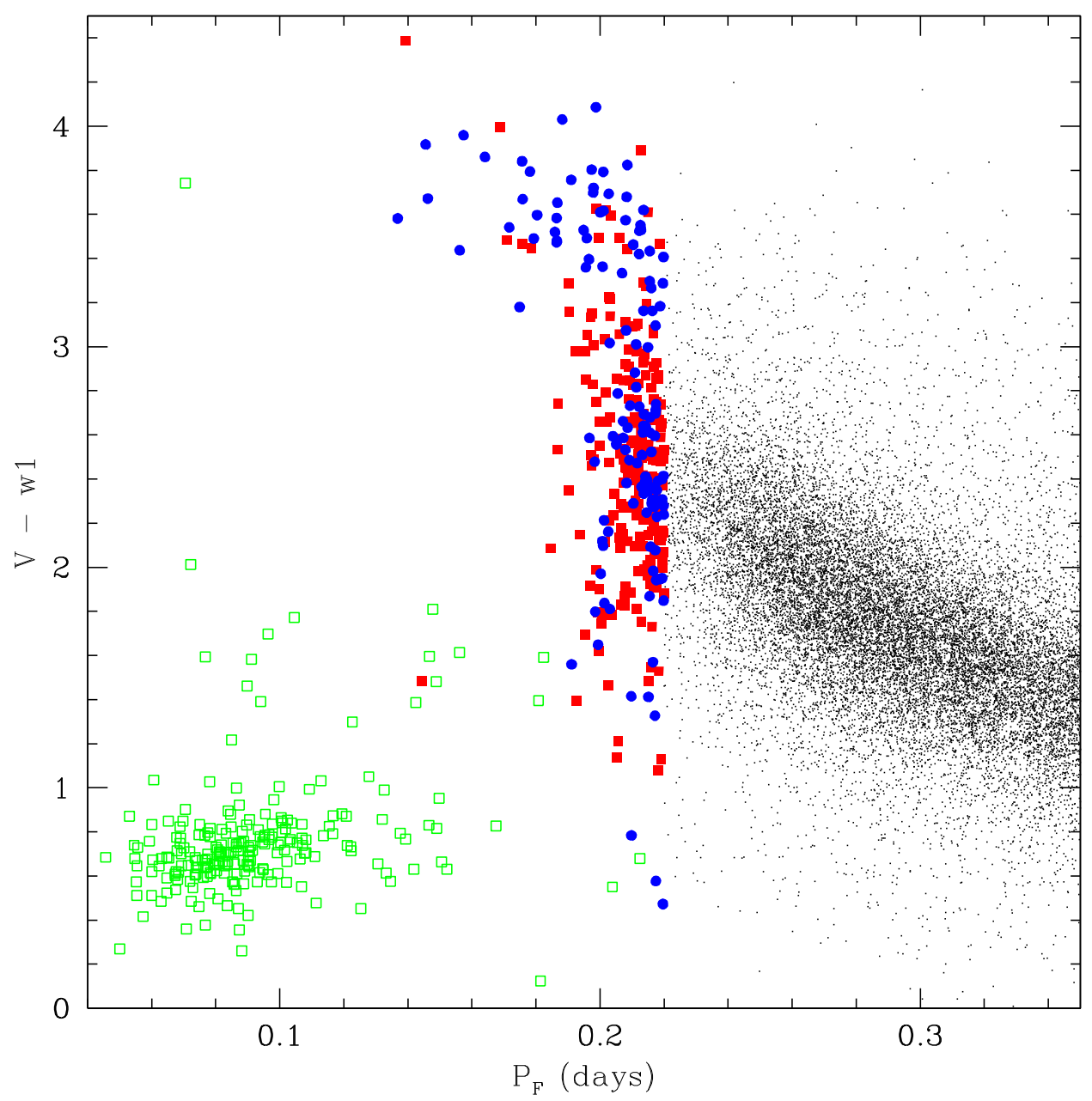

Figure 3. Period-color distribution for short-period variables. $\delta$ Scuti variables (green open boxes), ellipsoidal candidates (blue dots), short period eclipsing binaries (red boxes), general ellipsoidal and eclipsing binaries (black points) are shown.

(A color version of this figure is available in the online journal.)
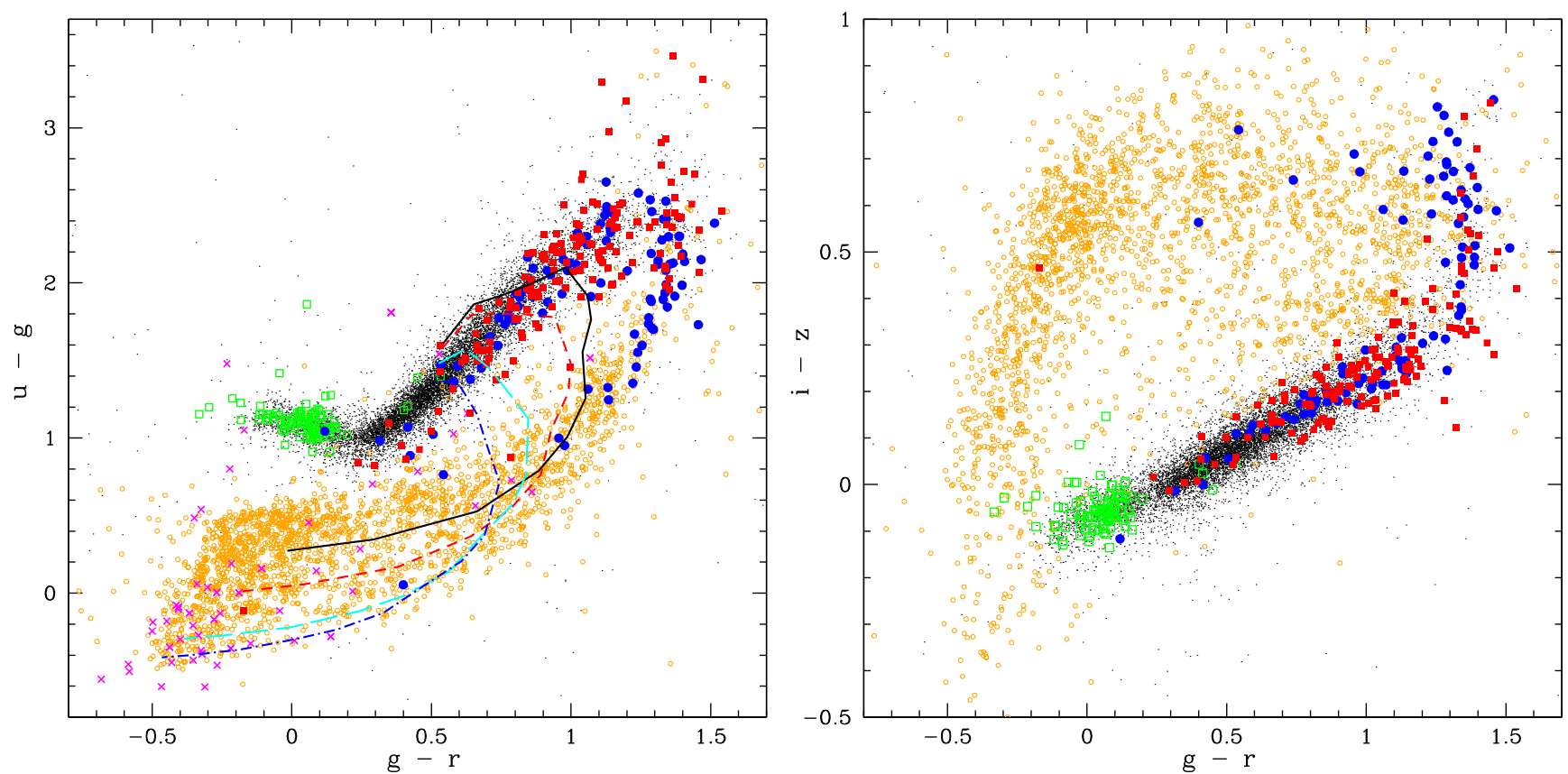

Figure 4. Optical color distribution for short-period variables. Colors are based on extinction correct SDSS photometry. The symbols are the same as Figure 3 with the addition of known CVs and white dwarfs shown as magenta crosses and spectroscopically selected WD+dM binaries from Rebassa-Mansergas et al. (2012) as small orange circles. The solid black line, short dashed red line, long dashed cyan line, and blue dash-dotted line give model colors of WDMS binaries where the WDs have effective temperatures of 10,000 K, 20,000 K, 30,000 K, and 40,000 K, respectively, from Rebassa-Mansergas et al. (2012).

(A color version of this figure is available in the online journal.) 

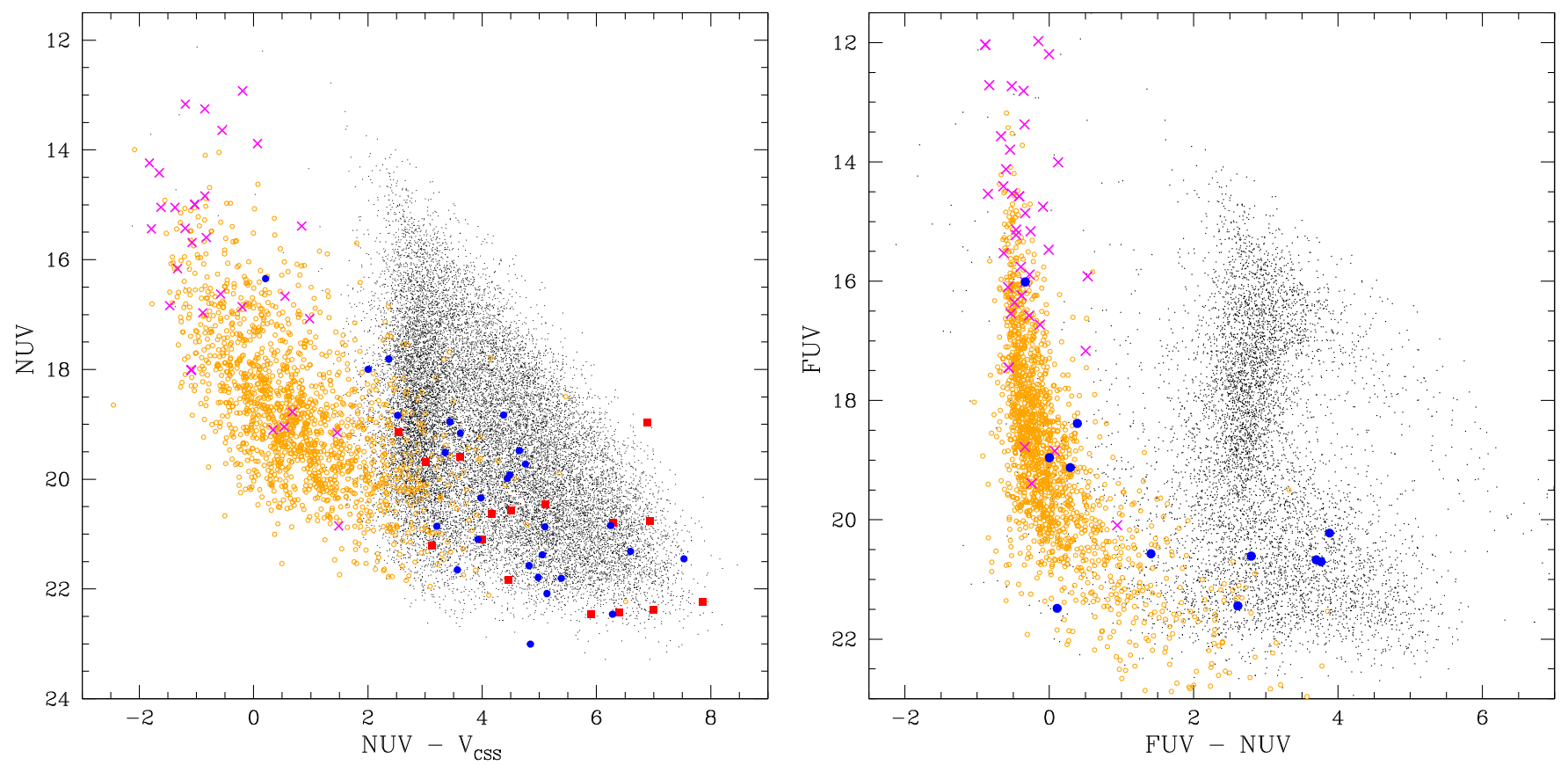

Figure 5. UV and optical colors of the periodic variable stars. In the left panel, we plot the GALEX GR5 near-UV colors of the periodic variables. The symbols match those given in Figure 4.

(A color version of this figure is available in the online journal.)

isochrones given by Rebassa-Mansergas et al. (2012) and colors of other known WDMS systems detected in CSDR1 by Drake et al. (2014).

Figure 4 also shows that many of the short-period ellipsoidal candidates, exhibit excess color in $g-r$ compared to main sequence eclipsing binaries. This excess is completely consistent with the colors of spectroscopically identified WD+dM binaries from Rebassa-Mansergas et al. (2012). However, the colors of most objects lie at the red end of the known distribution, where WDs cooler than $10,000 \mathrm{~K}$ are expected. Such systems are very difficult to identify spectroscopically since cool WDs can be much fainter than $M$ dwarfs. Thus they do not give rise to significant $u$-band flux. From $i-z$ we see that the ellipsoidal candidates are generally redder that the regular eclipsing sources, suggesting that the M dwarfs in WDMS pairs are usually later types than those in MS binary pairs. The excess $g-r$ color is indicative of a warm secondary, rather than a cool $\mathrm{M}$ dwarf. In contrast, we see that most of the objects with regular eclipsing light curves do not exhibit any $g-r$ color excess, suggesting that most are truly main-sequence pairs, although there may still be some confusion (as outlined below).

Aside from the true color features, we note that the $u$-band magnitudes of the redder candidates are likely to be affected to some extent by the SDSS red leak. ${ }^{11}$ There is also likely to be a measurable amount of scatter in color due to the SDSS data being taken at a single epoch.

In cases where one of the components is a hot WD, one expects the presence of significant UV flux. Therefore, we matched the periodic variables with GALEX sources (within $\left.5^{\prime \prime}\right)$. We found matches for 20,000 of the 61,000 sources in Drake et al. (2014) periodic variable catalog.

In Figure 5, we compare the ellipsoidal candidates and eclipsing sources, with the known WD binaries as noted earlier. The eclipsing and ellipsoidal systems are not well separated in NUV $-V_{\text {CSS }}$ color and overlap with other periodic variables

\footnotetext{
11 http://www.sdss3.org/dr9/imaging/caveats.php
}

(as do many of the spectroscopically WDMS systems). Some of the ellipsoidal candidates do stand out in FUV - NUV colors. However, very few are detected at the depth of GALEX FUV measurements. As expected, the short-period eclipsing systems are not detected in GALEX FUV data.

The GALEX results strongly suggest that the companions must be much cooler than most of the known WDMS systems. Since WDs are among the most common stars, it is expected that numbers of cool WD+dM binaries should exceed the number of hot systems (Rebassa-Mansergas et al. 2013). Low accretion-rate CVs and pre-CV systems, such as SDSS 121010.1+334722.9 (Pyrzas et al. 2012), may also produce very little emission with cool WDs. The detection of FUV emission from the ellipsoidal candidates strongly suggests that some fraction of these systems contain hot WDs.

Another piece of evidence suggesting that ellipsoidal candidates are not contact binaries comes from the fact that some of the light curves exhibit clear eclipses. In Figure 6, we plot examples of short-period systems where a compact source eclipses a distorted companion. The shallow depth of the eclipses, along with the short ingress and egress times, suggests that the eclipsing source is much more compact than the distorted companion. The light curves also lack any obvious secondary eclipse. This further suggests that compact eclipsing source is much fainter than the star undergoing the eclipse. Thus in these cases, at least, one of the components must be fainter and smaller than its companions.

The lower two light curves of Figure 6 demonstrate the difficulty there can be when discerning whether a system has the W-shaped eclipses of a contact binary or the sinusoidal light curve of an ellipsoidally distorted $\mathrm{M}$ dwarf with a faint, compact companion.

For most ellipsoidal candidates, there was no sign of distinct eclipses. It is possible that some of the companions could be neutron stars. Indeed, $\gamma$-ray pulsar PSR J2339-0533 (Romani $\&$ Shaw 2011) was among the periodic sources detected in our 


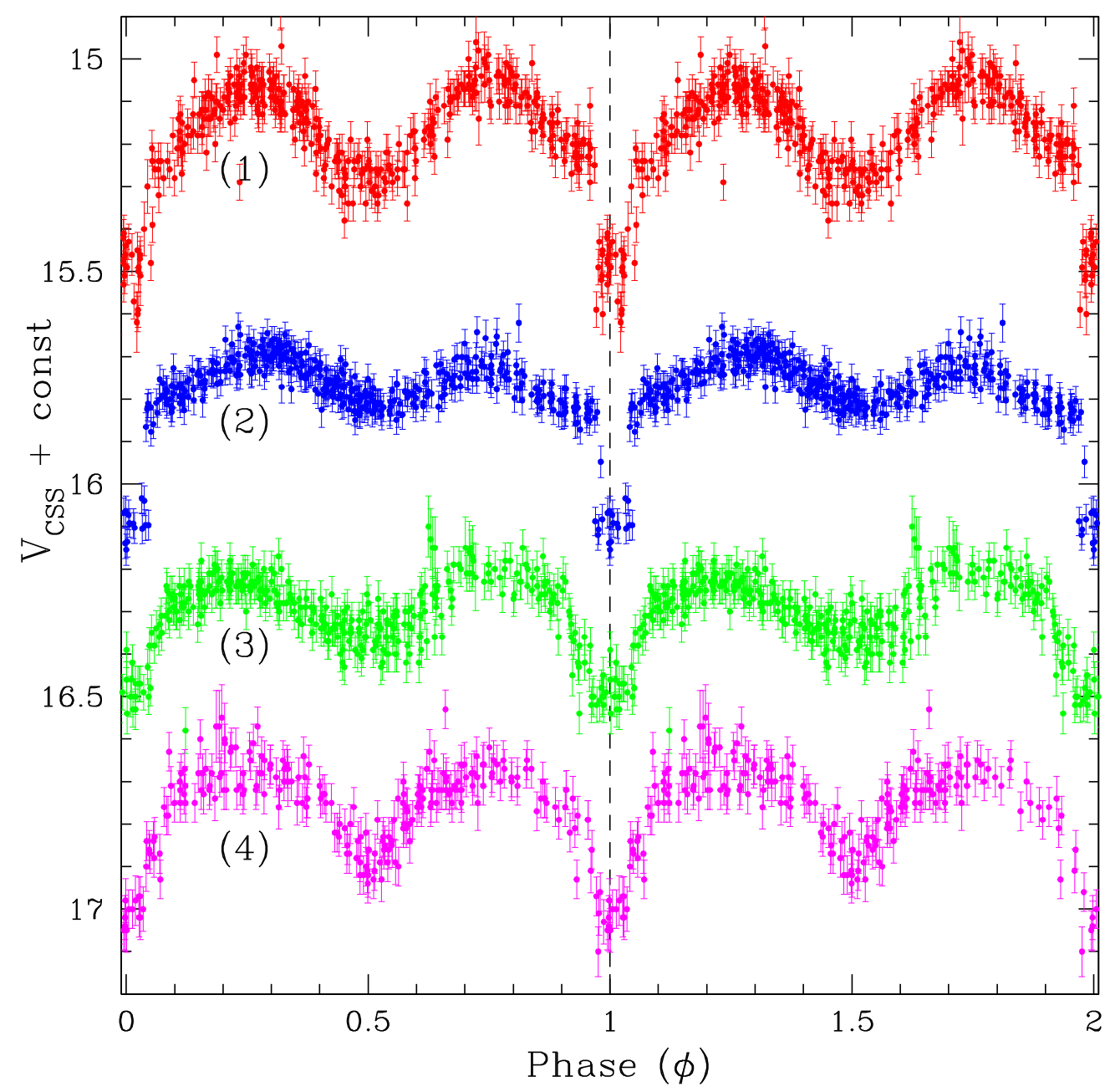

Figure 6. Candidate ellipsoidal variables with discrete eclipsing features (top three curves) compared to a morphologically similar, likely contact binary. The variables labeled 1-4 are CSS_J090826.3+123648 (red, $P=0.139$ day), CSS_J093508.0+270049 (blue, $P=0.201$ day), CSS_J165352.4+391410 (green, $P=0.220$ day), and CSS_J234131.5+375439 (magenta, $P=0.171$ day), respectively.

(A color version of this figure is available in the online journal.)

analysis of periodic variables in CSDR1. This pulsar exhibits a high level of variability due to a highly distorted companion. It also has a period in the range of these sources (0.193 day). We matched the short-period sources with unidentified sources in the Fermi-LAT catalog (Nolan et al. 2012) but found no convincing matches. In this short-period range, we expect that low-mass companions to neutron stars should give rise to a higher level of variability than observed, suggesting that most of systems are likely to be due to WDs rather than other compact sources.

\subsection{Spectroscopic Follow-up}

In order to confirm the nature of the ultra-short-period binary candidates, we carried out low-resolution spectroscopy with the Optical System for Imaging and Low Resolution Integrated Spectroscopy (OSIRIS) tunable imager and spectrograph (Cepa et al. 2003; Cepa 2010) at the $10.4 \mathrm{~m}$ Gran Telescopio CANARIAS (GTC), located at the Observatorio Roque de los Muchachos in La Palma, Canary Islands, Spain. The heart of OSIRIS is a mosaic of two $4 \mathrm{k} \times 2 \mathrm{k}$ e2 $\mathrm{v}$ CCD44-82 detectors that gives an unvignetted field of view of $7.8 \times 7.8 \mathrm{arcmin}^{2}$ with a plate scale of $0.127 \operatorname{arcsec}_{\text {pixel }}{ }^{-1}$. However, to increase the signal-to-noise ratio of our observations, we chose the standard operation mode of the instrument, which is a $2 \times 2$ binning mode with a readout speed of $100 \mathrm{kHz}$. All spectra were obtained with the OSIRIS R1000B grism. We used the 1.23 arcsec width slit, oriented at the parallactic angle to minimize losses due to atmospheric dispersion. The resulting resolution, measured on arc lines, was $R \sim 700$ in the approximate 3500-8000 $\AA$ spectral range. An additional seven spectra were obtained with the Palomar $5 \mathrm{~m}$ telescope (P200) using the Double Beam Spectrograph using a 1 arcsec-width slit and spectral range 3700-10000 A.

All the spectra were reduced using standard reduction procedures within IRAF. The GTC observing program concentrated on taking spectra of the short-period objects with contact binary light curves, while the Palomar program concentrated on sources with the shortest periods (which are mostly ellipsoidal candidates). In addition to these programs, we matched all the ultra-short-period binaries with objects having spectra within the SDSS Data Release 10 (SDSS DR10; Ahn et al. 2014). We found three SDSS matches to our eclipsing candidates and one to an ellipsoidal candidate.

In Table 1, we present the details of the SDSS, Palomar, and GTC spectra and in Figures 7-9, we present the GTC, Palomar, and SDSS spectra, respectively. Spectral types were derived by comparison to SDSS classifications. In particular, the $\mathrm{M}$ dwarfs were classified using the high signal-to-noise combined templates of Bochnaski et al. (2007). Overall, the 

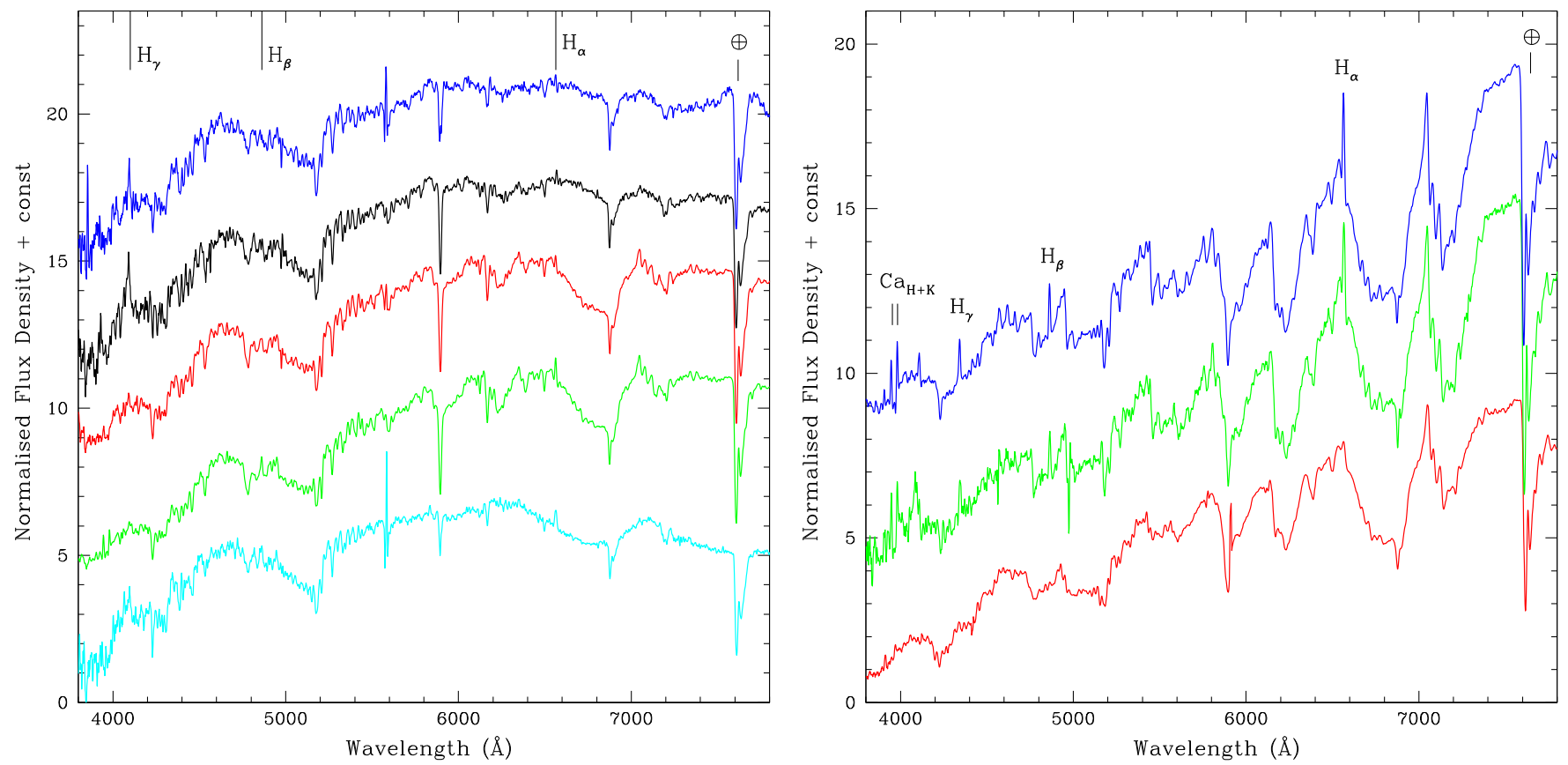

Figure 7. GTC optical spectra of ultra-short-period eclipsing binary candidates. In the left panel (from top to bottom), we plot the spectra for contact binaries with $\mathrm{K}$ types: CSS_J151531.4-153418 (blue), CSS_J044647.5+021635 (black), CSS_J234002.2+204122 (red), CSS_J145224.5+011522 (green), and CSS_J161945.9+241312 (cyan). In the right panel (from top to bottom), we plot the spectra for sources with M spectral types: CSS_J115533.4+354439 (blue), CSS_J213019.2-065136 (green), and CSS_J171508.5+350658 (red).

(A color version of this figure is available in the online journal.)

Table 1

Spectra of Ultra-short Period Binaries

\begin{tabular}{|c|c|c|c|c|c|c|c|c|c|}
\hline CRTS ID & R.A. & Decl. (J2000) & $V_{\text {CSS }}$ & Period & Telescope & Category & $u-g$ & $g-r$ & Spectral Type \\
\hline CSS_J090826.3+123648 & 09:08:26.26 & $+12: 36: 49.0$ & 15.27 & 0.1391985 & P200 & EA/ELL & 0.87 & 0.78 & M7V \\
\hline CSS_J111647.8+294602 & $11: 16: 47.83$ & $+29: 46: 02.8$ & 17.06 & 0.146249 & P200/SDSS & ELL & 1.73 & 1.27 & $\mathrm{M} 3.5 \mathrm{~V}^{\mathrm{b}, \mathrm{c}}$ \\
\hline CSS_J081158.6+311959 & $08: 11: 58.58$ & $+31: 19: 59.5$ & 16.13 & 0.156187 & P200 & ELL & 2.39 & 1.51 & $\mathrm{M} 2 \mathrm{~V}^{\mathrm{b}}$ \\
\hline CSS_J001242.4+130809 & 00:12:42.41 & $+13: 08: 09.6$ & 15.48 & 0.164086 & P200 & ELL & 1.78 & 1.29 & $\mathrm{M} 5 \mathrm{~V}^{\mathrm{b}}$ \\
\hline CSS_J112243.5+372130 & $11: 22: 43.44$ & $+37: 21: 30.2$ & 16.56 & 0.168744 & P200 & EW & 2.70 & 1.44 & $\mathrm{M} 4.5 \mathrm{~V}$ \\
\hline CSS_J041950.2-012612 & 04:19:50.16 & $-01: 26: 12.1$ & 17.87 & 0.175752 & P200 & EW & $\ldots$ & $\ldots$ & M3V \\
\hline CSS_J171508.5+350658 & $17: 15: 08.50$ & $+35: 06: 58.7$ & 16.56 & 0.178549 & P200/GTC & EW & 2.75 & 1.40 & M2V \\
\hline CSS_J112237.0+395219 & $11: 22: 37.06$ & $+39: 52: 19.9$ & 17.07 & 0.184749 & SDSS & EW & 1.51 & 0.62 & $\mathrm{~K} 2 \mathrm{~V}$ \\
\hline CSS_J090119.2+114254 & 09:01:19.22 & $+11: 42: 54.7$ & 16.34 & 0.1866878 & $\mathrm{P} 200$ & ELL & 0.95 & 0.98 & $\mathrm{M} 4 \mathrm{~V}^{\mathrm{b}}$ \\
\hline CSS_J151531.4-153418 & $15: 15: 31.43$ & $-15: 34: 18.6$ & 17.61 & 0.190148 & GTC & EW & & & $\mathrm{K} 5 \mathrm{~V}$ \\
\hline CSS_J101022.0+012438 & 10:10:22.01 & $+01: 24: 38.5$ & 18.8 & 0.190321 & P200 & EW & 2.34 & 1.38 & MOV \\
\hline CSS_J145224.5+011522 & $01: 32: 24.53$ & $+01: 15: 22.0$ & 15.04 & 0.196014 & GTC & EW & $\ldots$ & $\ldots$ & $\mathrm{K} 9 \mathrm{~V}$ \\
\hline CSS_J161945.9+241312 & $16: 19: 45.93$ & $+24: 13: 12.4$ & 17.86 & 0.19727 & GTC & EW & 1.91 & 1.04 & K6V \\
\hline CSS_J044647.5+021635 & $04: 46: 47.55$ & $+02: 16: 35.0$ & 18.03 & 0.19777 & GTC & EW & $\ldots$ & $\cdots$ & $\mathrm{K} 5 \mathrm{~V}$ \\
\hline CSS_J234002.2+204122 & $23: 40: 02.25$ & $+20: 41: 22.2$ & 16.29 & 0.198047 & GTC & EW & 2.76 & 1.33 & K7V \\
\hline CSS_J213019.2-065136 & $21: 30: 19.20$ & $-06: 51: 36.2$ & 16.42 & 0.198785 & GTC & EW & 2.24 & 1.38 & M2V \\
\hline CSS_J115533.4+354439 & $11: 55: 33.44$ & $+35: 44: 39.3$ & 15.77 & 0.199725 & GTC & EW & 2.11 & 1.34 & M2V \\
\hline CSS_J012119.1-001950 & $01: 21: 19.12$ & $-00: 19: 50.7$ & 15.70 & 0.207282 & SDSS & EW & 2.52 & 1.21 & K7V \\
\hline CSS_J122814.6+534746 & $12: 28: 14.61$ & $+53: 47: 46.7$ & 17.23 & 0.212048 & SDSS & EW & 2.25 & 1.08 & $\mathrm{~K} 5 \mathrm{~V}$ \\
\hline
\end{tabular}

Notes. Column 1: Catalina ID. Columns 2 and 3: right ascension and declination Column 4: period in days. Column 5: source of the spectrum where P200 is the Palomar $5 \mathrm{~m}$, and GTC is Gran Telescopio Canarias $10.4 \mathrm{~m}$. Column 6: type of periodic variable (ELL = ellipsoidal candidate, EW = contact or W UMa type, EA = detached or Algol type). Columns 7 and 8: extinction corrected colors from SDSS DR10 photometry. Column 9: spectral type.

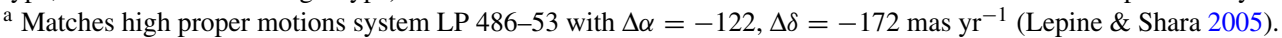

${ }^{\mathrm{b}}$ System where radial velocity variations were measured.

${ }^{c}$ Matches SDSS J111647.81+294602.7. Previously identified as candidate binary based on SDSS spectra by Clark et al. (2012).

GTC spectra consist of five K dwarfs and three early M dwarfs, while the shorter period systems observed with Palomar all contain M dwarfs. One system with an SDSS spectrum and one object observed by the GTC were re-observed with Palomar as a consistency check. The spectral types were in excellent agreement.
Inspection of the spectra revealed the presence of clear Balmer emission in almost all of the spectra. Such emission is seen in a large fraction of $\mathrm{M}$ dwarfs and increases significantly for late types (Bochnaski et al. 2005). However, only $\sim 10 \%$ of M1 and M2 stars are known to exhibit such activity. Furthermore, Balmer emission is also seen in the $\mathrm{K}$ dwarfs observed by the 

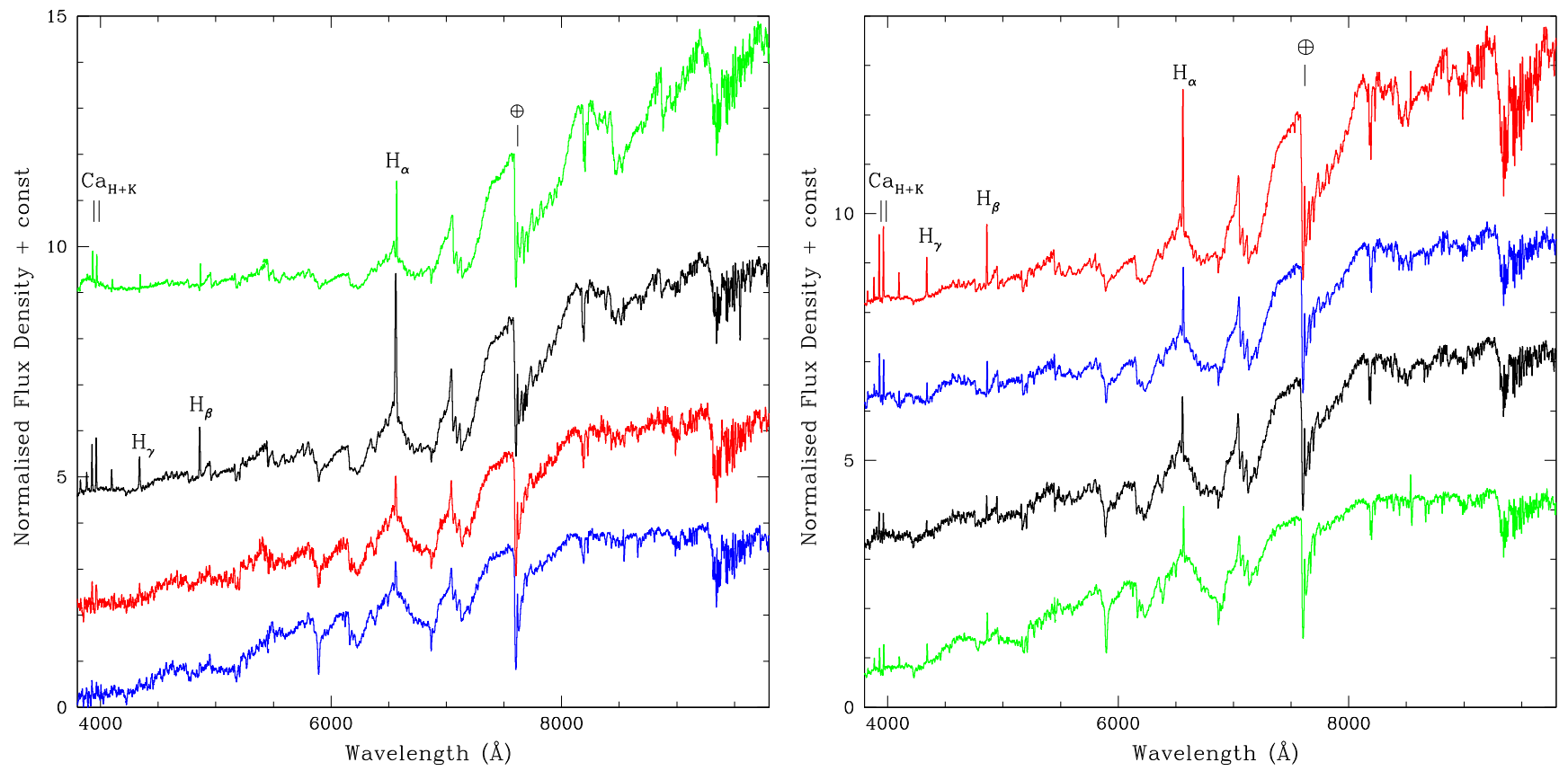

Figure 8. Palomar optical spectra of ultra-short-period eclipsing binary candidates. In the left panel (from top to bottom), we plot the contact binaries sources: CSS_J090826.3+123648 (LP 486-53, green), CSS_J112243.5+372130 (black), CSS_J041950.2-012612 (red), and CSS_J171508.5+350658 (blue). In the right panel (from top to bottom), we plot the ellipsoidal candidates: CSS_J001242.4+130809 (red), CSS_J090119.2+114254 (blue), CSS_J111647.8+294603 (SDSS J111647.81+294602.7, black), and CSS_J081158.6+311959 (green).

(A color version of this figure is available in the online journal.)

Table 2

Ultra-short-period Binary Fits

\begin{tabular}{|c|c|c|c|c|c|c|c|c|c|}
\hline CRTS ID & $\begin{array}{c}V 1_{\max } \\
\left(\mathrm{km} \mathrm{s}^{-1}\right)\end{array}$ & $\begin{array}{c}V 2_{\max } \\
\left(\mathrm{km} \mathrm{s}^{-1}\right)\end{array}$ & $\begin{array}{c}M 1 \\
\left(M_{\odot}\right)\end{array}$ & $\begin{array}{c}M 2 \\
\left(M_{\odot}\right)\end{array}$ & $\begin{array}{l}\text { Teff1 } \\
(\mathrm{K})\end{array}$ & $\begin{array}{l}\text { Teff } 2 \\
(\mathrm{~K})\end{array}$ & $\begin{array}{c}I \\
(\mathrm{deg})\end{array}$ & $\begin{array}{c}R 1 \\
\left(R_{\odot}\right)\end{array}$ & $\begin{array}{c}R 2 \\
\left(R_{\odot}\right)\end{array}$ \\
\hline CSS_J001242.4+130809 & 77 & 110 & 0.24 & 0.17 & 3200 & 2900 & 40.5 & 0.410 & $0.36^{\mathrm{a}}$ \\
\hline,,, & 101 & 225 & 0.58 & 0.22 & 6100 & 2900 & 70.9 & 0.034 & $0.34^{\mathrm{b}}$ \\
\hline CSS_J090119.2+114254 & 54 & 256 & 0.58 & 0.12 & 7800 & 3100 & 69.9 & 0.018 & 0.58 \\
\hline CSS_J090826.3+123648 & 86 & 294 & 0.62 & 0.18 & 6500 & 2500 & 85.9 & 0.008 & 0.26 \\
\hline CSS_J111647.8+294602 & 109 & 242 & 0.52 & 0.23 & 6500 & 3150 & 73.5 & 0.016 & 0.32 \\
\hline CSS_J081158.6+311959 & 101 & 206 & 0.47 & 0.23 & 6300 & 3400 & 61.3 & 0.018 & 0.33 \\
\hline
\end{tabular}

Notes. As noted in the text, these fits are based on velocities and fluxes from a single component in each system. They are thus only approximate. The fit temperatures for the secondary components are very uncertain and have been rounded to the nearest $100 \mathrm{~K}$. Column 1: Catalina ID. Columns 2 and 3: maximum radial velocities. Columns: 4 and 5: masses of components. Columns 6: fit effective temperatures of component. Column 7: input effective temperature based on observed spectral type. Column 8: orbital inclination. Columns 9 and 10: average radii.

${ }^{a}$ Main-sequence binary model solution for the system.

${ }^{b}$ WDMS binary model solution for the system.

GTC. Only a couple of percent of the stars are expected to exhibit emission (Zhao et al. 2013). The enhanced number here is a sign of magnetic activity induced in these compact binaries. Similar highly enhanced active fractions were found WD+dM systems by Morgan et al. (2012). Additionally, Ca H+K lines are present in emission in most of the spectra. This is also a sign of strong magnetic interaction between the components.

Comparison of the spectra for the ellipsoidal candidates with the regular eclipsing binaries does not show any obvious difference. Among the sources from Table 1, only CSS_J090119.2+114254 and CSS_J090826.3+123648 show some evidence for an additional component at the bluest wavelengths. As noted in Table 1, these two stars also have the bluest colors $(u-g<1$ and $g-r<1)$ of the candidates with SDSS photometry. The lack of clear evidence for a WD companion in the ellipsoidal systems is not unexpected since van den Besselaar et al. (2007), Rodriguez-Gil et al. (2009), Rebassa-Mansergas et al. (2010), Pyrzas et al. (2012), Parsons et al. (2013), and Rebassa-Mansergas et al. (2013) all present examples of WDMS spectra where evidence for the WD is not clearly discerned. The lack of a blue component in these spectra suggests that the sources causing the distortion are generally cooler than the hot WDs that have previously been found using SDSS spectra (Rebassa-Mansergas et al. 2012). The absence of a blue component is also expected based on the SDSS and GALEX colors presented earlier.

\subsubsection{Radial Velocities}

Since the spectra of the short-period systems poorly constrain cases where the primary is too faint to be seen, we undertook a program to better classify the binary systems using radial velocity variations. For short-period systems, an unseen yet relatively massive companion (such as a WD) is expected to produce large radial velocity variations in low-mass companion stars (such as $\mathrm{M}$ dwarfs). The presence of small velocity variations can also be used to exclude possible sources of contamination, such as pulsating stars. For example, $\delta$ Scuti 


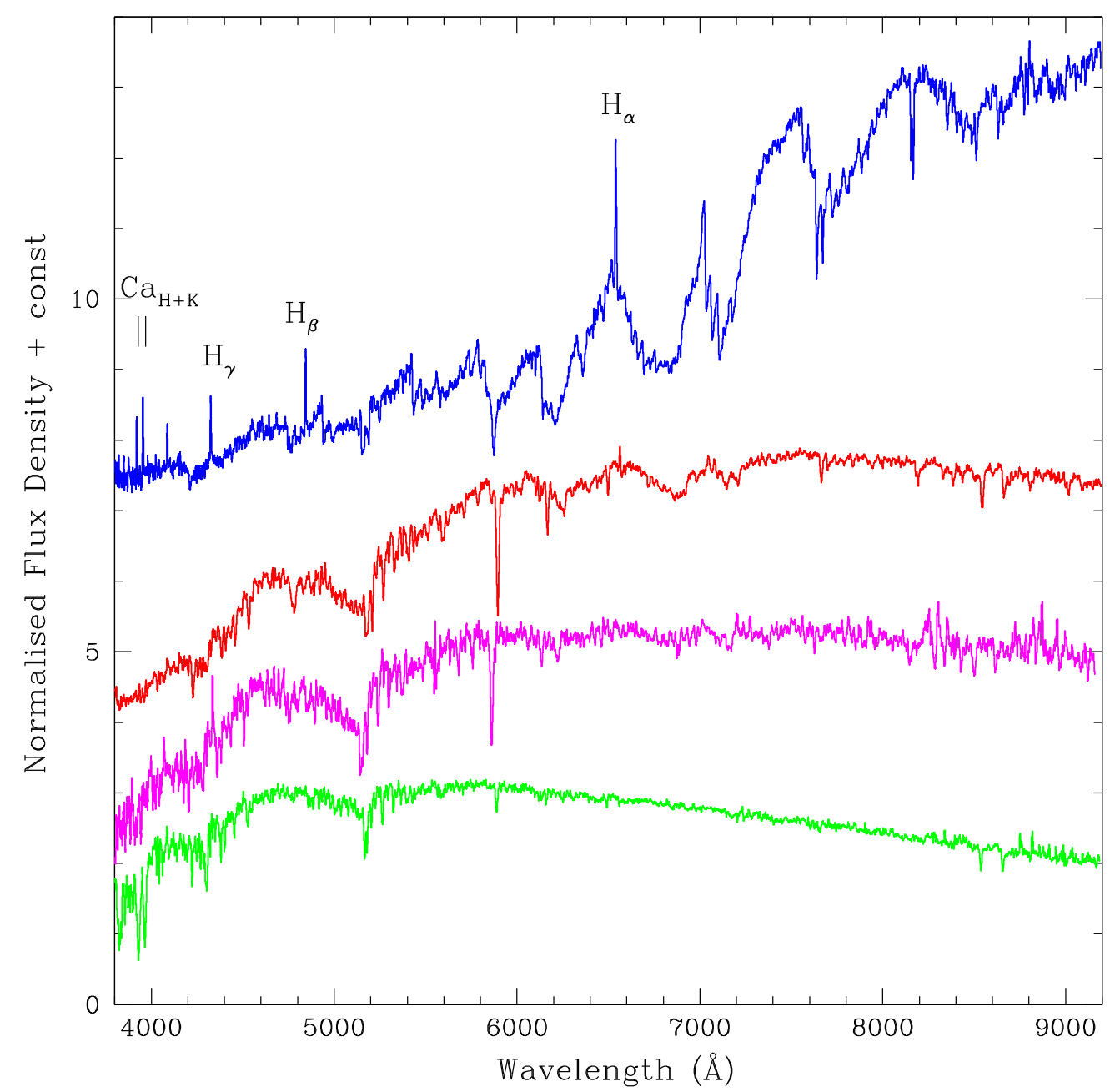

Figure 9. SDSS optical spectra of ultra-short-period eclipsing binary candidates. These spectra include (from top to bottom): CSS_J111647.8+294603 (blue), CSS_J112237.0+395219(red), CSS_J012119.1-001950 (magenta), and CSS_J122814.6+534746 (green).

(A color version of this figure is available in the online journal.)

variables have velocity variations of only a few $\mathrm{km} \mathrm{s}^{-1}$ (Penfold 1971; Zima et al. 2007; Antoci et al. 2013), while WD+dM binaries have typical radial velocity amplitudes of around $150 \mathrm{~km} \mathrm{~s}^{-1}$ (Nebot Gómez-Morán et al. 2011).

Multiple epochs of optical spectra were obtained for five of the systems noted in Table 1. As the spectra only exhibit clear emission from one component, it is only possible to measure the velocity variations of a single source in each system. Future high-resolution observations of these objects may enable the detection features from both sources.

For each source, we measured velocity variations by the average Doppler velocities of the Balmer and $\mathrm{Ca}$ II $\mathrm{H}+\mathrm{K}$ lines. In each case, the average was weighted by the relative strength of the lines. The velocity errors are for these low-resolution spectra are on the order of $\sim 15 \mathrm{~km} \mathrm{~s}^{-1}$. As the light curves themselves are measured over a period of years, the phases of our observations are expected to be accurate to $\sim 0.01$ cycle.

To determine the parameters for these binary systems, we simultaneously fit the observed radial velocities and light curves using the Nightfall ${ }^{12}$ software. In Table 2 , we present the fit parameters for the five systems and in Figures 10-12, we present the light curves and radial velocity variations. For each system,

${ }^{12}$ For details on Nightfall, see http://www.hs.uni-hamburg.de/DE/Ins/Per/Wichmann/Nightfall.html. we used the measured spectral type to estimate the approximate effective temperature based on Baraffe \& Chabrier (1996). The resulting binary system fits were sanity checked against radii and masses expected based on the updated models of $\mathrm{M}$ dwarfs presented Baraffe et al. (1998). Nevertheless, as only one component is observed in each binary, the system parameters in these fits are not very strongly constrained with the existing data. Observations of both sources would put much stronger constraints on the components. In particular, the temperatures of the WDs are very poorly constrained due to their lack of flux from these sources.

From the plots, it is clear that four out of five systems have velocity variations of $>100 \mathrm{~km} \mathrm{~s}^{-1}$. These four systems are most consistent with WD+dM binaries containing a cool WD with a low-mass M-dwarf companion. In fact, even without detection of lines from both sources, the radial velocities of the emission lines are only consistent with origin from a low-mass component. We can be certain that the lines we observed are not from objects in the usual WD mass range $\left(0.4-0.8 M_{\odot}\right)$.

For the fifth system, CSS_J001242.4+130809, we found two possible solutions to the binary configuration. This object exhibits relatively small radial velocities, suggesting that a massive companion is not necessary to produce the observed variations. The first solution for this system consists of a pair of over-contact $\mathrm{M}$ dwarfs orientated at a high inclination to 


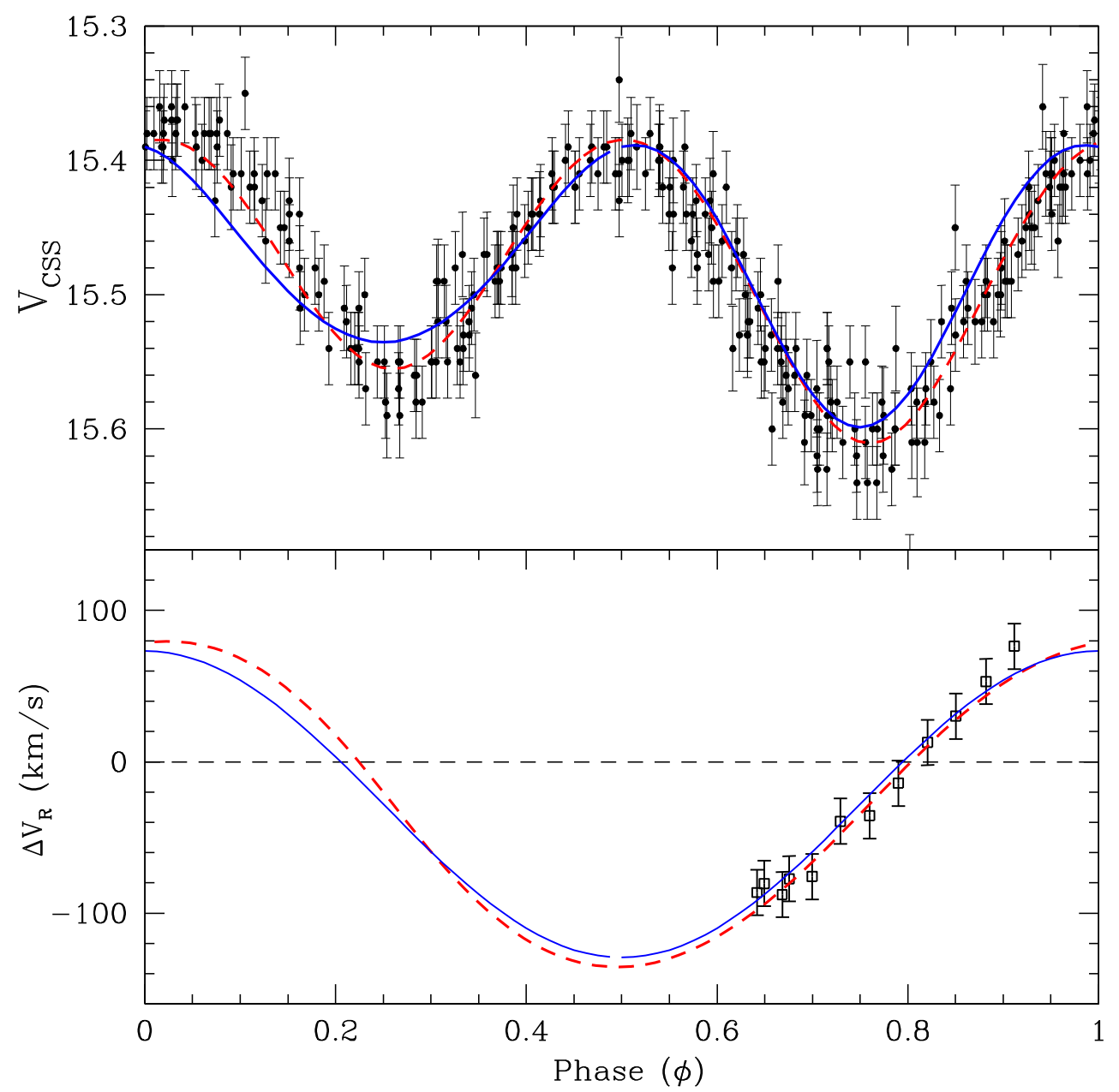

Figure 10. Light curve and radial velocities variations of CSS_J001242.4+130809. The red dashed lines give the dM+dM model fit to photometric and velocity variation. The blue lines show the best $\mathrm{WD}+\mathrm{dM}$ fit. The fits are uncertain since only a single set of spectral features is observed.

(A color version of this figure is available in the online journal.)
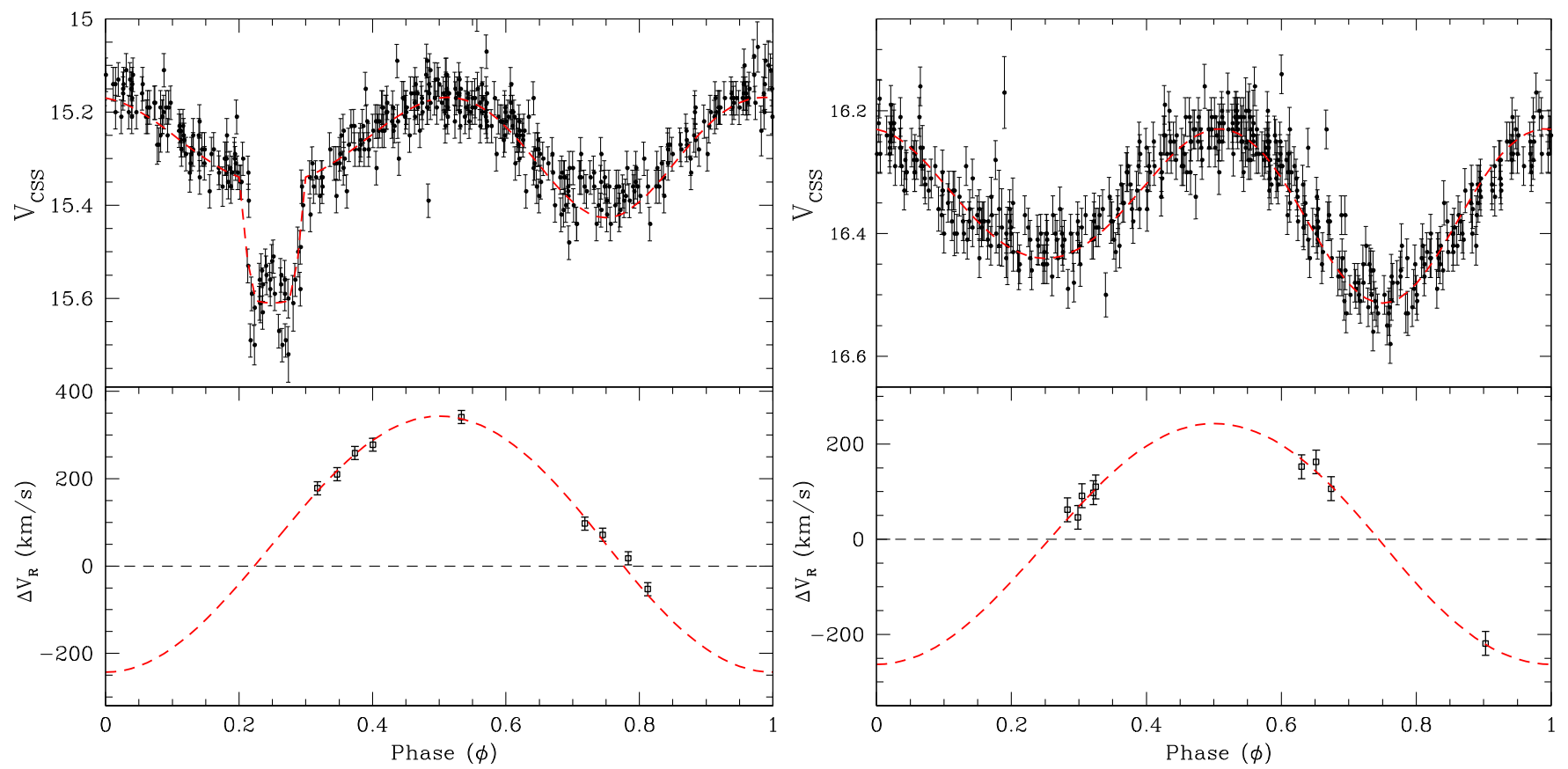

Figure 11. Light curves and radial velocities variations of ultra-short-period binaries. The left panel presents the results for CSS_J090826.3+123648, while the right panel shows the values for CSS_J090119.2+114254. The red dashed lines give the model fit to photometric and velocity variation. The fits are uncertain since only a single set of spectral features is observed.

(A color version of this figure is available in the online journal.) 

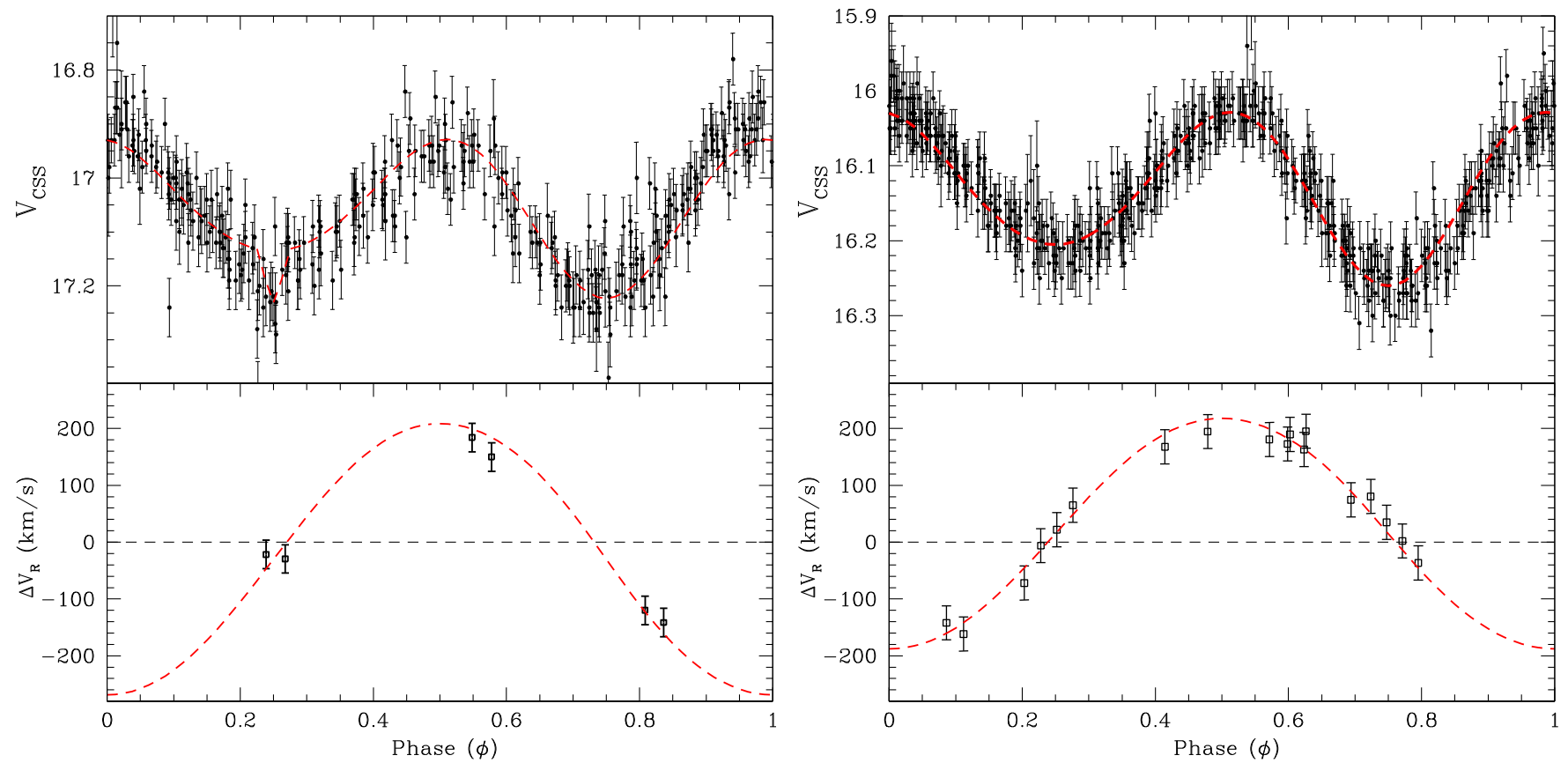

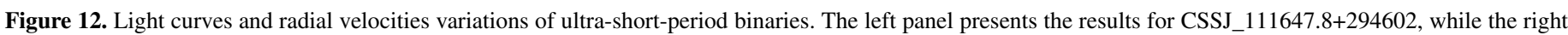

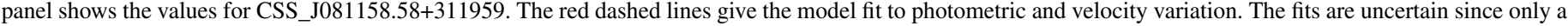
single set of spectral features is observed.

(A color version of this figure is available in the online journal.)

our line of sight (40.5). The second solution suggests that the photometric and radial velocity variations are due to a Roche lobe-filling M dwarf at a much smaller inclination (70.9). In the first model, the radial velocity variations seen in Balmer and $\mathrm{Ca}$ II emission lines would have to be due to the WD (unlike the other systems), while in the second model, a distorted M dwarf would give rise to the variations. The difference between the two fits can be seen from Figure 10. The goodness-of-fit value for the $\mathrm{dM}+\mathrm{dM}$ model was $\chi_{r}^{2}=1.1$, while for the WD+dM model it was $\chi_{r}^{2}=1.8$. The $\mathrm{dM}+\mathrm{dM}$ model is thus favored by this. Additionally, the presence of emission from the $\mathrm{M}$ dwarf seems more likely as it occurs for the other four systems. If the observed emission does originate from the WD, this would suggest that material from the M dwarf is being accreted onto the WD. For two similar WD+dM systems, van den Besselaar et al. (2007) and Bruch (1999) found that the emission was due to the activity of the M dwarf, rather than the WD. Alternately, there are a few known examples of very low-mass WDs in binaries (Brown et al. 2012). Future high signal-to-noise observations, with increased phase coverage and multi-band photometry, should be able to break the degeneracy between the two models.

\section{DISCUSSION AND CONCLUSIONS}

In Drake et al. (2014) we classified 231 of the sources as shortperiod eclipsing contact binaries and 136 as candidate ellipsoidal variables. Thus, among the $\sim 31,000$ contact and ellipsoidal binary candidates, there are $\sim 370$ systems with periods below the 0.22 day cutoff. Accounting for recent discoveries, this work still increases the number of ultra-short-period eclipsing binaries and short-period ellipsoidal systems by an order of magnitude. However, our analysis demonstrates the difficulty in separating ultra-short-period binaries containing pairs of late main-sequence stars from those with cool WDs in WDMS systems. For example, in cases where the observed flux from a WDMS system is dominated the main-sequence star, the presence of a WD may not be seen in optical spectra or multicolor photometry (such as provided by SDSS and GALEX). This suggests that such systems are best found using light curves and confirmed using radial velocity variations. However, in cases where the amplitude of variation is $>0.3 \mathrm{mag}$, systems are likely to contain main-sequence binaries since the variation amplitude of a WDMS is limited by the degree of distortion that the main-sequence star can undergo before transferring material onto the WD (Parsons et al. 2013).

The PTF survey discovered three WD+dM systems with cool WDs in a search for planets transiting $M$ dwarfs (Law et al. 2012). This survey covered $<500 \mathrm{deg}^{2}$ for systems reaching $m_{R} \sim 18$. The three systems found have periods between 0.35 and 0.45 days. Based on this result, the authors suggest that binaries with cool WDs are preferentially found at large orbital radii, in contrast to the systems with hotter WDs presented by Rebassa-Mansergas et al. (2012). However, since the search undertaken by Law et al. (2012) was designed to find planets transiting M dwarfs, they used the standard Box-Least Squares (BLS) technique of Kovacs et al. (2002) to find variables. The BLS technique is specifically designed to discover small dips in otherwise featureless light curves rather than other general types of periodic behavior. In particular, since short-period WD+dM systems exhibit large sinusoidal flux variations due to the distorted $\mathrm{M}$ dwarf, their light curves do not resemble transiting planets (Drake et al. 2013). Additionally, Parsons et al. (2013) have shown that only a small fraction of the hundreds of known WD+dM binaries exhibit the discrete eclipses that Law et al. (2012) were sensitive to. Considering this fact and the relatively small area covered by the Law et al. (2012) analysis compared to our survey, it is not surprising they did not find cool WD+dM systems with short periods. Indeed, in contrast to the Law et al. (2012) results, Pyrzas et al. (2012) discovered a cool $(6000 \mathrm{~K}) \mathrm{WD}+\mathrm{dM}$ system with a period of just 0.12 day. 
Nevertheless, as our analysis is limited to systems with periods of $<0.22$ day, we cannot constrain the possibility that there is indeed a much larger fraction of cool WD binaries at long periods.

The separation of WD+dM binaries from $d M+d M$ systems is more difficult when the $\mathrm{M}$ dwarfs are in the spectral range from M0V to M2.5V. Such M dwarfs have masses in the range of 0.6-0.3 $M_{\odot}$ (Baraffe \& Chabrier 1996) and absolute magnitudes of $8<M_{V}<11$ (Kroupa \& Tout 1997). Since their masses overlap with the WD mass distribution (Kleinman et al. 2013), they give rise to the same radial velocity amplitudes as WDMS systems. Additionally, in WDMS binary systems, one does not expect to observe split narrow emission lines that may be present in a main-sequence binary pairs, although pairs of lines may not be present in many such systems. Furthermore, as WDs have absolute magnitudes in the range of $12<M_{V}<16$ (Bergeron et al. 1995; Andreuzzi et al. 2002), cool WDs can be many magnitudes fainter than their M-dwarf companions and thus be undetectable. In order to distinguish these systems, one has to investigate slight variations between over-contact and WDMS binary solutions. However, if discrete eclipses are present, this demonstrates that these systems are detached rather than in contact.

In this analysis we have found many of the short-period sources with the ellipsoidal variable-type light curves. As noted above, while contact and detached binaries have different light curve shape, WD+dM binaries and over-contact binaries with amplitudes $<0.3 \mathrm{mag}$ often have very similar shapes. Some of the systems exhibit excess $g-r$ flux compared to main-sequence binaries. A few systems do exhibit FUV - NUV colors (similar to those of previously known $\mathrm{CVs}$ and WD+dM binaries). However, these binary systems generally do not exhibit different NUV $-V_{\text {CSS }}$ colors compared with main-sequence eclipsing binaries or other periodic variables. Such systems therefore cannot be found through color selections with SDSS or GALEX, nor with individual SDSS spectra as analyzed by RebassaMansergas et al. (2012).

The radial velocities for four of the five systems observed are sufficient to establish that the primaries are much more massive than the $M$ dwarfs seen in the spectra. Instead, they are consistent with cool WDs. For the remaining system, two situations are possible; a WD+dM binary or an M dwarf pair. An $\mathrm{M}$ dwarf pair is favored due to the improved model fit, relatively small radial velocities, and presence of narrow emission lines from the primary.

In comparison to our results, Nefs et al. (2012) present a number of short-period systems that exhibit small amplitudes and sinusoidal light curves. Of the four Nefs et al. (2012) sub0.2 day systems, the light curve morphology of 07g-3-05744 best matches that of the ellipsoidal type variables we select. Additionally, the extinction corrected SDSS DR9 colors for this source $(u-g=0.89, g-r=0.67, r-i=1.2, i-z=0.74)$ strongly suggest that the object has a color excess. This system appears to be more likely an WD+dM binary than a mainsequence pair. No spectroscopic observations were presented for this systems by Nefs et al. (2012). Two of the remaining three short-period objects from Nefs et al. (2012) have SDSS $u$-band uncertainties greater than a magnitude, while the third is not detected and none of these objects have measured radial velocities. This makes it difficult to completely discount the possibility that the systems contain cool WDs. However, this possibility seems very unlikely for two of the systems, since they exhibit regular detached binary light curves. In particular, 19h-3-14992 clearly exhibits two eclipses of similar span and differing depth. Nefs et al. (2012) confirms that this detached system contains an M dwarf based on a WHT ISIS spectrum. However, the spectrum itself does not cover the $\lambda<5000 \AA$ region where a WD might be seen, and there is no evidence for a companion in the spectrum presented.

Comparing the number of short-period binaries discovered by Nefs et al. (2012), one finds a much greater fraction than found in our analysis. For examples, Nefs et al. (2012) found 14 sources with periods of $<0.22$ day from a sample of 262,000 sources, while we find 367 among 198 million sources. This difference can be partially explained by the much greater red sensitivity of the Nefs et al. (2012) J-band data. In contrast, Lohr et al. (2013) found 49 similar short-period binaries among 30 million light curves using very well-sampled, but shallower, SuperWASP data. Another reason for the relatively large number of Nefs et al. (2012) sources is that the survey fields were taken much closer to the Galactic plane. The CSS project avoided the plane because of crowding, yet as shown by Drake et al. (2014), the contact binaries are strongly concentrated near the Galactic plane (as expected).

Using GTC, SDSS, and Palomar spectroscopy, we have confirmed the presence of M-dwarf systems among ultra-shortperiod eclipsing contact binaries. The only other spectroscopically confirmed ultra-short-period M-dwarf contact binary was discovered by Davenport et al. (2013). This system, originally identified by Becker et al. (2011), has a period of $\sim 0.2$ day and clearly exhibits a sinusoidal light curve with an amplitude of 0.2 mag. As with our ellipsoidal variable selection, this object exhibits a slight deviation from a single sinusoid. The object's light curve shape is most consistent with our confirmed WD+dM systems. Furthermore, as with the WD+dM binaries, the Davenport et al. (2013) spectra lack evidence for a blue component yet exhibit a single $\mathrm{H} \alpha$ emission line. Davenport et al. (2013) find the masses of the components to be $M_{1}=0.54 M_{\odot}$ and $M_{2}=0.25 M_{\odot}$. As noted above, such masses are consistent with a either an early $\mathrm{M}$ dwarf or WD primary. The strongest evidence against this system being a WD+dM system is the presence of faint pairs of Ca I absorption lines at $6102 \AA$ and $6122 \AA$. However, as Pyrzas et al. (2012) have shown, WD+dM binaries also include metal lines in their spectra. In such cases, the source of the lines has been attributed to WD accretion of M-dwarf wind. Since Davenport et al. (2013) did not attempt to fit a WD+dM model, the exact nature of this systems still appears uncertain.

Our analysis firmly shows the existence of a population of contact binaries with periods of $<0.2$ day. This result suggests that current binary evolution models discounting the existence of these systems provide an incomplete picture of the binary population. Nevertheless, since M dwarfs are very common and short-period systems very rare, it is entirely possible that such systems only occur under special conditions. One possibility suggested by Nefs et al. (2012) is that such systems occur due to interactions in stellar triple systems. Such interactions may not be uncommon since, as noted by Rucinski et al. (2007), hierarchical triples are very common among shortperiod binaries.

The recent theoretical results of Jiang et al. (2012) suggest that contact binaries are not found with masses of less than $0.61 M_{\odot}$ and periods of $<0.2$ day due to very short evolutionary times $(<1$ Gyr) that are caused by unstable mass transfer. It is not possible to resolve the timescale of the unstable transfer presented by Jiang et al. (2012), though the presented results 
suggest this is $<<0.01 \mathrm{Gyr}$. It is possible that the small number of short-period contact binaries we detect might be those undergoing such transfer. However, there is no observational evidence for this within the light curves.

In stark contrast to Jiang et al. (2012), the Stepien (2006) binary models explain the lack of such systems as being due to such systems not reaching contact within a Hubble time. Jiang et al. (2012) explains the difference between their model and that of Stepien (2006) as being due to a different assumption for the angular momentum loss rate. Given the apparently highly discrepant theories, the cause for the contact binary period limit remains very poorly understood. Nevertheless, our discovery of contact systems below the 0.2 day limit should serve as an additional constraint for future binary models.

In the near future, the Gaia mission (Perryman et al. 2001) and the Variables in the Via Lactea (VVV) survey (Minniti et al. 2010) will begin to harvest Galactic disk fields and are expected to find millions of periodic variables (Eyer et al. 2012; Catelan et al. 2013). Although Gaia is only expected to reach stars to the same depth as CSDR1, with far fewer epochs, it is expected to have ultra-precise photometry. This will greatly increase the accuracy with which over-contact and WD+dM systems can be separated. Likewise, the LSST survey will reach far greater depths than any existing wide-field survey (Ivezic et al. 2008). The LSST will thus be able to probe M-dwarf binaries within a far greater volume than other surveys, and thereby enable us to better constrain the true frequency and period distribution of such systems.

CRTS and CSDR1 are supported by the U.S. National Science Foundation under grant AST-1313422. The CSS survey is funded by the National Aeronautics and Space Administration under grant No. NNG05GF22G issued through the Science Mission Directorate Near-Earth Objects Observations Program. J.L.P. acknowledges support from NASA through Hubble Fellowship grant HF-51261.01-A awarded by the STScI, which is operated by AURA, Inc. for NASA, under contract NAS 526555. Some of this work is based on observations made with the Gran Telescopio CANARIAS (GTC), installed in the Spanish Observatorio del Roque de los Muchachos of the Instituto de Astrofsica de Canarias on the island of La Palma. Support for M.C. and G.T. is provided by the Ministry for the Economy, Development, and Tourism's Programa Inicativa Científica Milenio through grant IC120009, awarded to Millennium Institute of Astrophysics (MAS), Santiago, Chile; by Proyecto Basal PFB-06/2007; and by Proyecto FONDECYT Regular Nos. 1110326 and 1141141. SDSS-III is managed by the Astrophysical Research Consortium for the Participating Institutions of the SDSS-III Collaboration Funding for SDSS-III has been provided by the Alfred P. Sloan Foundation, the Participating Institutions, the National Science Foundation, and the U.S. Department of Energy Office of Science. The SDSS-III Web site is http://www.sdss3.org/.

\section{REFERENCES}

Ahn, C. P., Alexandroff, R., Allende, P., et al. 2014, ApJS, 211, 17 Andreuzzi, G., Richer, H. B., Limongi, M., \& Bolte, M. 2002, A\&A, 390, 961 Antoci, V., Handler, G., Grundahl, F., et al. 2013, MNRAS, 435, 1563 Baraffe, I., \& Chabrier, G. 1996, ApJL, 461, L51
Baraffe, I., Chabrier, G., Allard, F., \& Hauschildt, P. H. 1998, A\&A 337,403

Becker, A. C., Bochanski, J. J., Hawley, S. L., et al. 2011, ApJ, 731, 17

Bergeron, P., Wesemael, F., \& Beaucamp, A 1995, PASP, 107, 1047

Bertin, E., \& Arnouts, S. 1996, A\&AS, 117, 393

Bochnaski, J. J., Hawley, S. L., Reid, I. N., et al. 2005, AJ, 130, 1871

Bochnaski, J. J., West, A. W., Hawley, S. L., \& Covey, K. R. 2007, AJ, 133,531

Brown, W., Kilic, M., Prieto, C. A., \& Kenyon, S. J. 2012, ApJ, 744, 142

Bruch, A. 1999, AJ, 117, 3031

Catelan, M., Minniti, D., Lucas, P. W., et al. 2013, in Regional Variable Star Conference, 40 Years of Variables Stars: A Celebration of Contributions by Horace A. Smith, ed. K. Kinemuchi et al. (Ann Arbor, MI: Physics \& Astronomy Department, Michigan State Univ.), arXiv:1310.1996

Cepa, J. 2010, Highlights of Spanish Astrophysics V, Astrophysics and Space Science Proceedings (Berlin: Springer), 15

Cepa, J., Aguiar-González, M., Bland-Hawthorn, J., et al. 2003, Proc. SPIE, 4841, 1739

Clark, B. M., Blake, C. H., \& Knapp, G. R. 2012, ApJ, 744, 119

Davenport, J. R. A., Becker, A. C., West., A. A., et al. 2013, ApJ, 764, 62

Dimitrov, D. P., \& Kjurkchieva, D. P. 2010, MNRAS, 406, 2559

Drake, A. J. 2003, ApJ, 589, 1020

Drake, A. J. 2006, AJ, 131, 1044

Drake, A. J., Beshore, E., Djorgovski, S. G., et al. 2012, BAAS, 219, 428.20

Drake, A. J., Catelan, M., Djorgovski, S. G., et al. 2013, ApJ, 763, 32

Drake, A. J., Djorgovski, S. G., García-Álvarez, D., et al. 2014, ApJS, 213, 9

Eyer, L., Palaversa, L., Mowlavi, M., et al. 2012, Ap\&SS, 341, 207

Ivezic, Z., Tyson, J. A., Acosta, E., et al. 2008, arXiv:0805.2366

Jiang, D., Han, Z., Ge, H., Yang, L., \& Li, L. 2012, MNRAS, 421, 2769

Kleinman, S. J., Kepler, S. O., Koester, D., et al. 2013, APJS, 204, 5

Kovács, G., Zucker, S., \& Mazeh, T. 2002, A\&A, 391, 369

Kroupa, P., \& Tout, C. A. 1997, MNRAS, 287, 402

Law, N. M., Kraus, A. L., Street, R., et al. 2012, ApJ, 757, 133

Lepine, S., \& Shara, M. M. 2005, AJ, 129, 1483

Lohr, M. E., Norton, A. J., Kolb, U. C., et al. 2012, A\&A, 542, 124

Lohr, M. E., Norton, A. J., Kolb, U. C., et al. 2013, A\&A, 549, 86

Maceroni, C., \& Rucinski, S. M. 1997, PASP, 109, 782

Minniti, D., Lucas, P. W., Emerson, J. P., et al. 2010, NewA, 15, 433

Morgan, D. P., West, A. A., Garces, A., et al. 2012, AJ, 144, 93

Nebot Gómez-Morán, A., Gänsicke, B. T., Schreiber, M. R., et al. 2011, A\&A, 536,43

Nefs, S. V., Birkby, J. L., Snellen, I. A. G., et al. 2012, MNRAS, 425, 950

Nolan, P. L., Abdo, A., Ackermann, M., et al. 2012, ApJS, 199, 31

Norton, A.J, Payne, S. G., Evans, T., et al. 2011, A\&A, 528, 90

Norton, A. J., Wheatley, P. J., West, R. G., et al. 2007, A\&A, 467, 785

Parsons, S. G., Gänsicke, B. T., Marsh, T. R., et al. 2013, MNRAS, 429, 256

Penfold, J. E. 1971, PASP, 83, 497

Perryman, M. A. C., de Boer, K. S., Gilmore, G., et al. 2001, A\&A, 369, 339

Pojmanski, G. 1997, AcA, 47, 467

Pyrzas, S., Gänsicke, B. T., Brady, S., et al. 2012, MNRAS, 419, 817

Rebassa-Mansergas, A., Gänsicke, B. T., Rodŕguez-Gil, P., et al. 2010, MNRAS, 402, 620

Rebassa-Mansergas, A., Nebot Gómez-Morán, A., Schreiber, M. R., et al. 2012, MNRAS, 419, 806

Rebassa-Mansergas, A., Zorotovic, M., Schreiber, M. R., et al. 2013, MNRAS, 433, 3398

Rodriguez-Gil, D. R., Torres, M. A. P., Gänsicke, B. T., et al. 2009, A\&A, 496, 805

Romani, R. W., \& Shaw, M. S. 2011, ApJL, 743, L26

Rucinski, S. M. 1992, AJ, 103, 960

Rucinski, S. M. 1997, MNRAS, 382, 393

Rucinski, S. M. 2001, AJ, 122, 1007

Rucinski, S. M. 2006, MNRAS, 368, 1319

Rucinski, S. M., Pribulla, R., \& van Kerkwijk, M. H. 2007, AJ, 134, 2353

Southworth, J. 2012, in Proc. Orbital Couples: Pas de Deux in the Solar System and the Milky Way, ed. F. Arenou \& D. Hestroffer (Paris: Observatorie de Paris), 51

Stepien, K. 2006, AcA, 56, 347

van den Besselaar, E. J. M., et al. 2007, A\&A, 466, 1031

Zhao, J. K., Oswalt, T. D., Zhao, G., et al. 2013, AJ, 145, 140

Zima, W., Lehmann, H., Sttz, Ch., Ilyin, I. V., \& Breger, M. 2007, A\&A, 471,237 